\title{
Functional Uncoupling of Inhibitory Interneurons Plays an Important Role in Short-Term Sensitization of Aplysia Gill and Siphon Withdrawal Reflex
}

\author{
Louis-Eric Trudeau and Vincent F. Castellucci \\ Laboratoire de Neurobiologie et Comportement, Institut de recherches cliniques de Montréal, Montréal, Québec H2W 1R7 \\ and Centre de recherches en sciences neurologiques, Université de Montréal, Montréal, Québec H3C 3J7, Canada.
}

Attempts to explain learning-associated potentiation of synaptic transmission in model systems such as withdrawal reflexes in the mollusk Aplysia or the hippocampus of vertebrates have focused on the mechanisms by which transmitter release is increased in the principal elements of the circuit. Increased transmission in neuronal networks such as the gill and siphon withdrawal reflex (GSWR) of Aplysia may, however, also be caused by a decrease of transmitter release by inhibitory interneurons. The importance and function of cholinergic inhibitory transmission in the GSWR network were investigated. Central application of the nicotinic cholinergic antagonist $d$-tubocurarine (d-TC) considerably potentiated gill contractions, evoked either by nerve stimulation or by tactile stimulation of the siphon. Compound EPSPs evoked in motoneurons upon siphon nerve stimulation were also significantly prolonged following application of d-TC, but were unaffected by hexamethonium, a blocker of excitatory ACh receptors in Aplysia. Recordings from excitatory interneurons showed that they received excitation followed by powerful inhibitory input upon stimulation of the siphon nerve. Application of d-TC completely blocked this rapid inhibition, thus prolonging the compound EPSPs evoked in the interneurons. These effects were obtained at a concentration of $\mathrm{d}-\mathrm{TC}(100 \mu \mathrm{M})$ that almost totally blocked fast inhibitory cholinergic transmission, but was without effect on monosynaptic connections between sensory neurons and motoneurons of the reflex. Facilitation of (1) compound EPSCs in motoneurons and (2) evoked excitatory interneuronal firing was reduced in preparations already disinhibited by pretreatment with d-TC. Facilitation of sensory-motor synapses, however, was not reduced in the presence of d-TC, indicating that facilitatory interneurons are still activated under cholinergic blockade. These data show that transmission through the GSWR neuronal network is gated by a feedback inhibitory mechanism. They also suggest that a reduction of cholinergic inhibition onto excitatory inter-

\footnotetext{
Received July 6, 1992; reviscd Sept. 22, 1992; accepted Nov. 19, 1992.

We thank M. Klein, T. Ouimet, and L. Bernier for comments on the manuscript, I. Morin for preparing the illustrations, and N. Guay for typing the manuscript. This work was funded by Grant MA-10047 from the Medical Rescarch Council of Canada, and by the Richard and Edith Strauss Canada Foundation. L.-E.T. is the recipient of a "1967" Scholarship from the Conseil de Recherches en Sciences Naturclles et Génie du Canada.

Correspondence should be addressed to Vincent F. Castellucci, Laboratoire de Neurobiologie et Comportement, Institut de recherches cliniques de Montréal, 110 ouest, avenue des Pins, Montréal, Québec. H2W 1R7, CAN.

Copyright (c) 1993 Society for Neuroscience 0270-6474/93/132126-10\$05.00/0
}

neurons may be a mechanism through which transmission within the GSWR network is increased during various forms of learning, such as sensitization. These data place new emphasis on the important role of inhibitory interneurons in determining the plastic properties of neuronal networks, in both invertebrates and vertebrates.

[Key words: Aplysia, inhibition, ACh, nicotinic receptor, sensitization, neuronal network, learning, reflex]

Most of the efforts aimed at understanding the neuronal modifications underlying short- and long-term behavioral changes associated with learning in Aplysia californica have been directed at the monosynaptic junctions between a subset of sensory neurons and identified motoneurons mediating the reflex behaviors that have been studied. That work has identified, at these critical sensory-motor synapses, cellular and molecular mechanisms that may partially account for the changes in behavioral output associated with learning and memory in this invertebrate (Castellucci et al., 1970; Brunelli et al., 1976; Hochner et al., 1986a,b; Montarolo et al., 1986; Dash et al., 1990; Bailey et al., 1992; Mayford et al., 1992).

We have recently presented evidence for the importance of the polysynaptic component of the neuronal network in the mediation and plasticity of the gill and siphon withdrawal reflex (GSWR) (Trudeau and Castellucci, 1992). Interneurons interposed between sensory and motor neurons act to amplify the sensory message reaching the motoneurons. Only a limited subset of interneurons of this type are presently known (Hawkins et al., 1981 a,h; Byrne, 1983; Frost et al., 1988). The polysynaptic network, consisting of both excitatory and inhibitory interneurons, appears to be an important site of plastic changes associated with sensitization of the GSWR. We have found that two neuromodulators of the reflex, 5-HT and small cardioactive peptide $\mathrm{B}\left(\mathrm{SCP}_{\mathrm{B}}\right)$, have differential effects on the neuronal network, the first preferentially potentiating the monosynaptic pathway, the second preferentially potentiating the polysynaptic pathway (Trudeau and Castellucci, 1992). Sensitization of the reflex by the activation of endogenous facilitator neurons may thus involve, to a large extent, a modification of synaptic transmission at the interneuronal level. The excitatory interneurons may become more excitable, they may increase their transmitter release onto motoneurons, or they may receive a decreased input from inhibitory interneurons. An examination of these possibilities and an analysis of the mechanisms involved will increase our understanding of the learning-associated modifications in this model system. 
In other systems, inhibitory interneurons have been suggested to be potentially important sites of modifications associated with learning. It has been proposed that the interplay between excitatory and inhibitory interneuronal activity may be a critical determinant of transmission in the neuronal networks controlling feeding in Pleurobranchaea californica (Davis et al., 1983) and phototaxis in Hermissenda (Lederhendler et al., 1986), in the Mauthner cell system of teleosts (Korn et al., 1992), and in the olfactory bulb network (Elaagouby et al., 1991) as well as in the hippocampal circuitry of the rat (Moot et al., 1991).

In Aplysia, little is known about the involvement of inhibitory transmission in neuronal network plasticity. In the GSWR network, it has been shown that transmission from L30 inhibitory interneurons to L29 facilitatory interneurons is reduced by the stimulation of fibers coursing through the left pleuro-abdominal connective (Frost et al., 1988), a procedure that usually induces sensitization of the reflex. Because the importance of these connections in the normal mediation of the reflex has not been evaluated, and because, in these experiments, the effect of conncctive stimulation on the output of the whole network was not monitored in parallel, the question of the involvement of inhibitory transmission in the plasticity of the GSWR remains unanswered.

In this article we have attempted to characterize further the role of the polysynaptic component of the GSWR neuronal network in the mediation and plasticity of the reflex. We have sought to address two issues. First, we wanted to know the importance of inhibitory transmission in the normal mediation of the GSWR. We have taken advantage of the fact that most described inhibitory synaptic transmission of the fast type in Aplysia nervous system is attributable to a $\mathrm{Cl}^{-}$conductance increase induced by $\mathrm{ACh}$ binding to nicotinic-type receptors. We were thus able to block this type of transmission by the use of the nicotinic cholinergic receptor blocker $d$-tubocurarine (dTC) (Tauc and Gerschenfeld, 1962; Blankenship et al., 1971; Kehoe, 1972), and to assess the effects of this blockade on the GSWR. Because we observed that such cholinergic inhibition was indeed significant and appeared to be involved in a feedback pathway, the second objective was to evaluate the involvement of disinhibitory processes in short-term sensitization of the reflex. Decreased inhibition upon excitatory interneurons could be responsible for increased excitatory transmission in the network. We thus compared facilitation of the reflex in normal versus disinhibited preparations. We found that although facilitation is still possible in disinhibited networks, it is considerably reduced. We therefore propose that a major mechanism of sensitization of the gill and siphon withdrawal reflex may involve a reduction of inhibitory cholinergic transmission.

\section{Materials and Methods}

Preparations. Aplysia californica (100-300 gm) were obtained from Marinus Inc. (Venice, CA) and housed individually in small compartments within a large aquarium containing artificial seawater (ASW; Forty Fathoms). The temperature was kept at $14^{\circ} \mathrm{C}$. Before dissection, animals were anesthetized with an injection of isotonic $\mathrm{MgCl}_{2}$ corresponding approximately to a third of their volume. Dissection was then carried out in a solution made with equal parts of isotonic $\mathrm{MgCl}_{2}$ and ASW. For the isolated ganglion preparation, the abdominal ganglion was dissected out and a long section of both pleuro-abdominal connectives and siphon nerve were kept intact. The ganglion was then exposed for 20 sec to $0.5 \%$ glutaraldehyde to kill muscle cells, and pinned to the bottom of a small $2 \mathrm{ml}$ Sylgard-coated chamber. The connective tissue covering the ganglion was removed with fine forceps. ASW was then perfused for $1 \mathrm{hr}$ before the beginning of experiments. A semi-intact preparation was used in some experiments; it consisted of the intact gill connected to the abdominal ganglion by the branchial nerve and the intact siphon connected to the abdominal ganglion through the siphon nerve; in other experiments only the siphon nerve was kept without the siphon. The abdominal ganglion was then isolated from the rest of the preparation with a small chamber that allowed nerves and connectives to exit through Vaseline-sealed apertures. This allowed independent applications of drugs to the ganglion. In these preparations, the gill was cannulated and continuously perfused with ASW. Gill contractions were measured through the output of a photoelectric cell above which the gill was placed. Stimuli were adjusted to evoke a moderate gill contraction (about $30 \%$ of maximum). Tactile stimulations of the siphon were delivered by an electromechanical stimulator. The siphon as well as the base of the gill were held in place by very small insect pins. In such preparations, the gill contractions evoked by tactile stimulation of the siphon may be artificially prolonged because of the involvement of reafference due to the siphon contracting against the pins. Because the same results were obtained with direct stimulation of the siphon nerve, this issue does not appear to have been a problem under our experimental conditions. The semi-intact preparations did not include an intact ctenidial (genital) nerve or an intact peripheral pathway, which are known to also play a role in the mediation of evoked gill contractions in the intact animal (Carew et al., 1974; Lukowiak and Peretz, 1977). We have restricted our analysis to the central component of the GSWR network and to its output through the branchial nerve (gill component). All experiments were performed at room temperature $\left(22^{\circ} \mathrm{C}\right)$.

Experiments were also carried out on an excitatory cholinergic synapse evoked in neuron R15 of the abdominal ganglion by threshold stimulation of an axon coursing in the right pleuro-abdominal connective (Frazier et al., 1967). Additional experiments were performed on identified cholinergic synapses in the buccal ganglion between neurons B4 or B5 and neurons B3 or B6 (Tauc and Gerschenfeld, 1962; Gardner, 1971; Baux et al., 1990). The postsynaptic neurons possess acetylcholine-gated $\mathrm{Cl}^{-}$channels and were voltage clamped at $-80 \mathrm{mV}$. Changes in $\mathrm{Cl}^{-}$reversal potential during experiments were always monitored and responses were expressed in conductance.

Electrophysiology. Intracellular electrodes were filled with either $3 \mathrm{M}$ $\mathrm{KCl}$ or $2 \mathrm{M} \mathrm{K}$-acetate and had a resistance between 1.5 and $15 \mathrm{M} \Omega$. Voltage recording and two-electrode voltage clamping were performed with an Axoclamp-2A (Axon Instruments) amplifier. Extracellular application of $\mathrm{ACh}$ to the cell body of neurons was performed by iontophoresis with an Axoprobe-1A (Axon Instruments) amplifier. In the microelectrode, $\mathrm{ACh}$ was dissolved in distilled water at a concentration of $1 \mathrm{M}$. Data were stored directly on hard disk as well as on VHS tape, and were analyzed with the SPIKE software (Hilal Associates). The area of postsynaptic responses was measured from the beginning of the postsynaptic potential/postsynaptic current (PSP/PSC) to the point where the PSP/PSC returned to its initial level. Data in the text are presented as mean \pm standard error of the mean.

Drugs and solutions. $d$-Tubocurarine chloride and hexamethonium chloride were obtained from Sigma (St. Louis, MO) and dissolved in ASW. Acetylcholine chloride was obtained from Anachemia (Toronto, Canada). The normal artificial seawater solution (ASW) contained, in $\mathrm{mM}, \mathrm{NaCl}, 460 ; \mathrm{MgCl}_{2}, 30 ; \mathrm{MgSO}_{4}, 25 ; \mathrm{CaCl}_{2}, 11 ; \mathrm{KCl}, 10 ;$ and HEPES buffer, 10, pH 7.6. The high-cation medium, containing 2.2 times the normal concentration of $\mathrm{Mg}^{2+}$ and 1.25 times the normal concentration of $\mathrm{Ca}^{2+}(2: 1 \mathrm{ASW})$, consisted of, in $\mathrm{mM}, \mathrm{NaCl}, 368 ; \mathrm{MgCl}_{2}, 101 ; \mathrm{MgSO}_{4}$, $20 ; \mathrm{CaCl}_{2}, 13.8 ; \mathrm{KCl}, 8$; and HEPES buffer, 10 , pH 7.6.

\section{Results}

Effect of d-tubocurarine on monosynaptic cholinergic transmission

To establish the importance of cholinergic transmission in the GSWR neuronal network, the effect of the nicotinic cholinergic receptor blocker d-TC was evaluated on evoked gill contractions and evoked compound EPSPs in motoneurons of the reflex. The drug was first tested on identified cholinergic synapses in Aplysia central ganglia to determine the concentration necessary to obtain complete blockade of nicotinic-type receptors. In preliminary experiments we confirmed that concentrations higher than $50 \mu \mathbf{M}$ were necessary to produce almost complete blockade (Tauc and Gerschenfeld, 1962; Blankenship et al., 1971; Kehoe, 


\section{A. BUCCAL SYNAPSE}

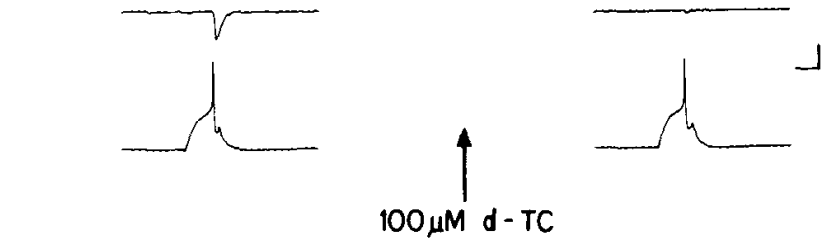

B. R15 SYNAPSE

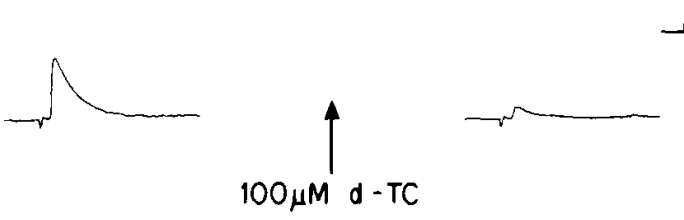

C.

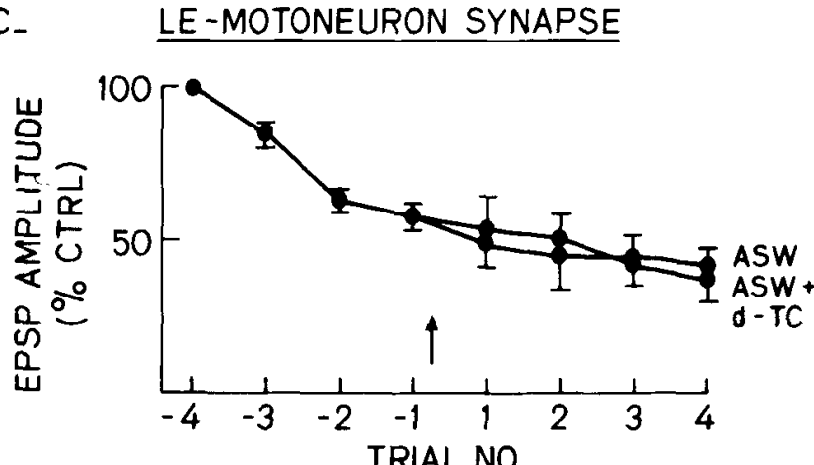

Figure 1. Effect of d-TC on synaptic transmission at identified cholinergic synapses. $A$, IPSCs, produced by $\mathrm{ACh}$-gated $\mathrm{Cl}$ " channels, were evoked in neurons $\mathrm{B} 3 / \mathrm{B} 6$ of the buccal ganglion by an action potential in neurons B4/B5. These responses were almost totally blocked by bathapplied d-TC $(100 \mu \mathrm{M})$. Calibration: $200 \mathrm{nS}-15 \mathrm{mV}$, $65 \mathrm{msec}$. $B$, An EPSP, produced by ACh-gated cationic channels, was evoked in neuron R15 of the abdominal ganglion by threshold stimulation of the right pleuro-abdominal connective. More than $75 \%$ of the peak amplitude was blocked by $100 \mu \mathrm{m}$ d-TC. Calibration: $4 \mathrm{mV}, 100 \mathrm{msec}$. $C$, EPSPs were evoked in motoneurons of the GSWR by an action potential in an $L E$ sensory neuron (interstimulus interval $=1 \mathrm{~min}$ ). The spontaneous depression of successive EPSPs was not altered by bath-applied d-TC following the fourth EPSP (arrow).

1972). At an identified cholinergic inhibitory synapse in the buccal ganglion, bath-applied d-TC $(100 \mu \mathrm{M})$ blocked more than $90 \%$ of the peak conductance measured in voltage-clamped B3/ B6 postsynaptic neurons upon an action potential evoked in a B4/B5 presynaptic neuron $(93.8 \pm 1.9 \%$ blockade; $n=4)$ (Fig. $1 A)$. The drug was also tested at an excitatory cholinergic synapse measured in abdominal ganglion neuron R15 upon threshold stimulation of the left pleuro-abdominal connective (Frazier et al., 1967). At a concentration of $100 \mu \mathrm{M}$, bath-applied d-TC blocked $75 \%$ of the peak EPSP amplitude $(75.5 \pm 6.9 \% ; n=3)$ (Fig. 1B). At this concentration, the drug would thus be expected to block most fast excitatory and inhibitory nicotinic cholinergic transmission within the GSWR neuronal network. Excitatory transmission between sensory and motoneurons of the GSWR is known not to be cholinergic (Segal and Koester, 1982). The effect of d-TC was nonetheless evaluated at these synapses in order to ascertain that the drug had no nonspecific effects. Sensory neurons of the LE cluster were stimulated intracellularly through a bridge circuit every minute and EPSPs were recorded

\section{A. SIPHON NERVE STIMULATION}
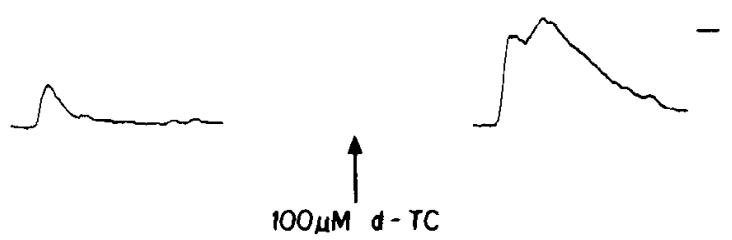

B. TACTILE SIPHON STIMULATION

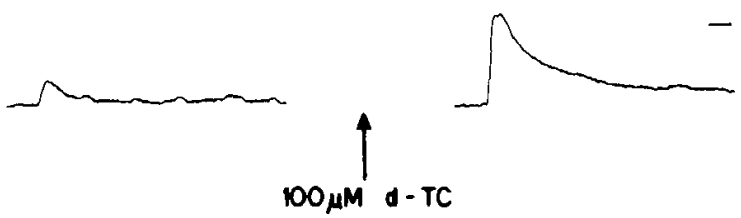

Figure 2. Effect of blocking central cholinergic transmission on evoked gill contraction in a semi-intact preparation. Gill contractions were measured through the output of a photoelectric cell on which the gill was placed. Central application (see Materials and Methods) of $100 \mu \mathrm{M}$ d-TC induced a considerable potentiation of contractions evoked either by direct siphon nerve stimulation $(A)$ or by tactile stimulation of the siphon skin $(B)$. Interstimulus interval was $10 \mathrm{~min}$. Control trace on the left, peak effect in the presence of d-TC on the right. Calibration, 1 sec.

in $\mathrm{L} 7$ (gill) or LFS (siphon) motoneurons of the abdominal ganglion. Four control responses were measured before bath application of $100 \mu \mathrm{M} \mathrm{d}-\mathrm{TC}$. In the control group, no drug was applied and the control decline of the EPSP was assessed. Figure $1 C$ shows that d-TC had no significant effect on LE-motoneuron synapses (control $n=4$; treated $n=4$ ) (see also Fig. $3 B$ ).

\section{Effect of d-tubocurarine on gill contractions}

We first began to characterize the overall involvement of cholinergic interneurons on transmission within the GSWR neuronal network. The drug d-TC $(100 \mu \mathrm{M})$ was applied on the abdominal ganglion and its effects were assessed on evoked gill contractions measured with a photoelectric cell (see Materials and Methods). The experiment was performed in five different preparations. In three of these, gill contractions were evoked with brief electrical shocks applied to the siphon nerve through a suction electrode (two shocks of $3 \mathrm{msec}$ duration, separated by $150 \mathrm{msec}$ ). In the other two preparations, tactile electromechanical stimulation of the siphon skin was used (two 50 msec taps, $9 \mathrm{~Hz}$ ). Three to five control contractions were recorded before application of d-TC in the small chamber isolating the abdominal ganglion from the rest of the preparation. The restricted central application of d-TC was necessary to prevent any effects due to the blockade of peripheral cholinergic receptors of the gill (Weiss et al., 1984). Interstimulus interval was $10 \mathrm{~min}$. ACh receptor blockade induced a marked increase in the size of gill contractions in all experiments (Fig. $2 A, B$ ). A mean increase of $421.9 \pm 137.4 \%$ above control was obtained when the area of the last response before exposure to d-TC was compared to that of the maximal response in presence of the $\operatorname{drug}(t=4.9, p<0.01 ; n=5)$.

\section{Effect of cholinergic blockade on compound EPSPs in motoneurons}

The receptor blocker was then tested on compound EPSPs evoked in LFS (siphon) or L7 (gill) motoneurons upon brief electrical 
A_

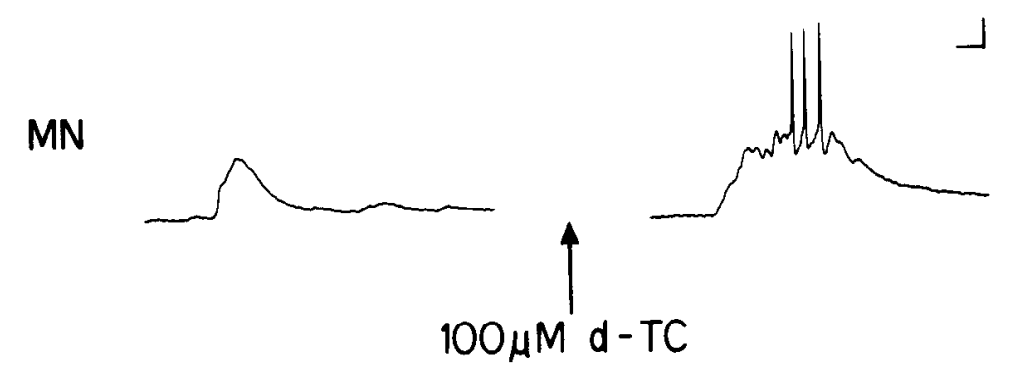

B.

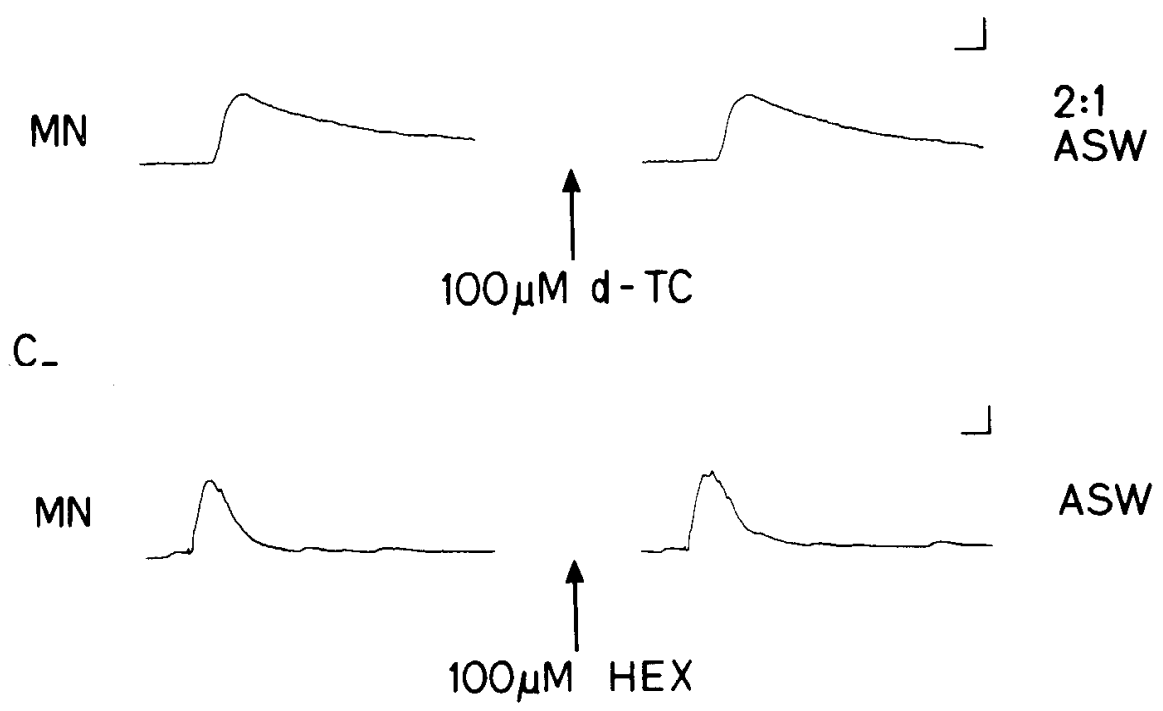

ASW

Figure 3. Effect of d-TC and hexamethonium on compound EPSPs evoked in motoneurons upon stimulation of the siphon nerve. $A$, In normal extracellular medium $(A S W), \mathrm{d}-\mathrm{TC}(100$ $\mu \mathrm{M})$ induced a potentiation of the excitatory input recorded in motoneurons $(M N)$ following electrical stimulation of the siphon nerve. Interstimulus interval was $5 \mathrm{~min}$. Calibration: $15 \mathrm{mV}, 70$ msec. $B$, In a high-divalent-cation medium $(2: 1 \mathrm{ASW})$, where polysynaptic transmission is greatly reduced, d-TC $(100 \mu \mathrm{M})$ had no significant effect on compound EPSPs. Calibration: $11 \mathrm{mV}$, $95 \mathrm{msec} . C$, In normal extracellular medium $(A S W)$, application of hexamethonium $(100 \mu \mathrm{M})$, a nicotinic blocker specific for cationic $\mathrm{ACh}$ receptors in this preparation, had no significant effect on compound EPSPs recorded in motoneurons. Calibration: $10 \mathrm{mV}, 95$ msec. stimulation of the siphon nerve through a suction electrode. Because in this and subsequent experiments similar results were obtained for the two types of motoneurons, the data were pooled. The stimulation was adjusted in order to evoke EPSPs with a peak amplitude between 15 and $30 \mathrm{mV}$ in motoneurons polarized to $-80 \mathrm{mV}$. We have shown that approximately $75 \%$ of the area of such responses is due to interneurons intercalated between sensory and motor neurons (Trudeau and Castellucci, 1992). Responses were measured every 5 min in normal ASW. Following six to eight responses to allow the EPSP to stabilize, d-TC was bath applied. Figure $3 A$ illustrates one EPSP before and one after drug application from a representative experiment. The initial peak amplitude of compound EPSPs was not significantly altered by the treatment $(7.1+5.0 \%$ increase $)$, but the following excitation was greatly prolonged, often giving rise to a second, distinct wave of excitation. Even though the neurons were current clamped to $-80 \mathrm{mV}$, the second wave of excitation often produced action potential firing. Similar results were obtained in nine other preparations. The mean increase in EPSP area (maximal effects) was $189.1 \pm 37.9 \%$ above control $(t=$ $4.9, p<0.01 ; n=10$ ). These results strongly suggested that inhibitory cholinergic interneurons normally play an important role in controlling excitatory interneuronal firing evoked by siphon afferent input. If the effect of d-TC was to block inhibitory transmission selectively at the interneuronal level, one would expect the drug to have no effect on compound EPSPs evoked in motoneurons under conditions where the polysynaptic contribution is minimized. Expcriments were therefore performed on compound EPSPs recorded with the abdominal ganglion bathed in a modified ASW containing a high concentration of divalent cations (2:1 ASW; see Materials and Methods) (Trudeau and Castellucci, 1992), which blocks most polysynaptic transmission by increasing action potential threshold in interneurons. Under such conditions, application of d-TC $(100 \mu \mathrm{M})$ had no significant effect on compound EPSPs (Fig. $3 B ; n=4$ ). A $9.3 \pm 6.9 \%$ increase in EPSP area was measured.

Because d-TC blocks both excitatory and inhibitory $\mathrm{ACh}$ receptors, the effects observed on compound EPSPs could also be indirectly caused by decreased cholinergic excitation in the neuronal network. This was tested with the use of hexamethonium, a drug known to block cxcitatory $\mathrm{ACh}$ receptors in Aplysia without affecting cholinergic receptors coupled to $\mathrm{Cl}$ " channels (Tauc and Gerschenfeld, 1962; Blankenship et al., 1971; Kehoe, 1972). At a concentration of $100 \mu \mathrm{M}$, we found that compound EPSPs evoked in motoneurons upon siphon nerve stimulation were totally unaffected. The mean, nonsignificant change in EPSP area was a decrease of $4.6 \pm 4.4 \%(n=4$; Fig. $3 C)$.

Effect of cholinergic receptor blockade on compound EPSPS in excitatory interneurons

The results presented above suggested that cholinergic inhibitory interneurons control the excitability of excitatory interneurons by an inhibitory feedback loop. Excitatory interneurons are thus expected to receive a direct wave of inhibition following excitatory input from the sensory neurons. Recordings were therefore obtained directly from excitatory interneurons. Neurons were impaled close to ink gland motoneurons L14, a region of the ventral surface of the abdominal ganglion that is known 
A.

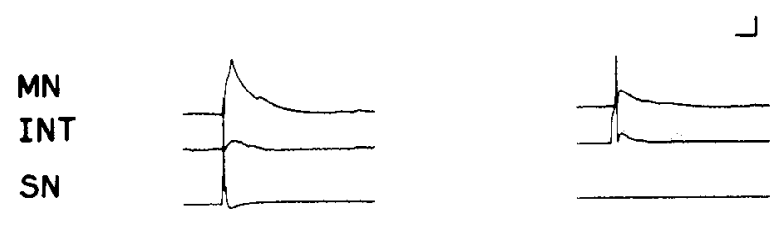

B.

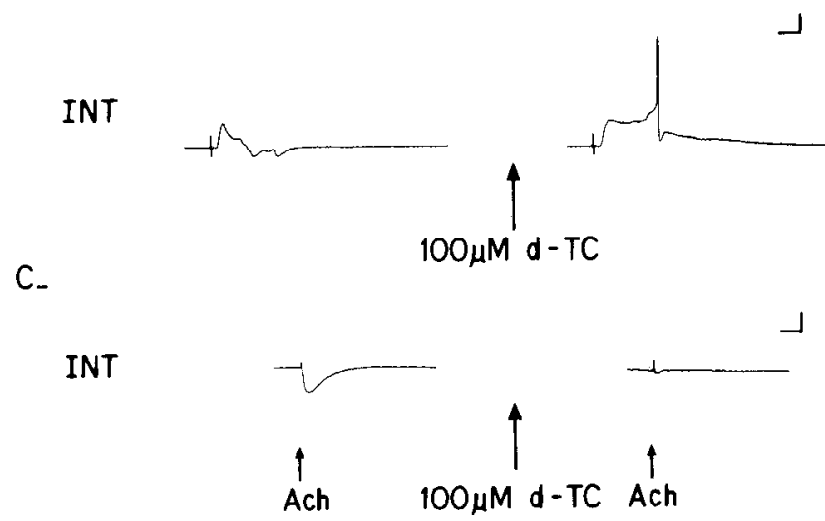

Figure 4. Cholinergic inhibitory input to excitatory interneurons. A Excitatory interneurons (INT) were identified by their synaptic connections. These receive a monosynaptic EPSP from sensory neurons of the LE cluster $(S N)$ (left), and in turn produce an EPSP in motoneurons $(M N)$ of the LFS type (right). Calibration for the left traces: $15 \mathrm{mV}-5$ $\mathrm{mV}-20 \mathrm{mV}, 85 \mathrm{msec}$; for the right traces: $15 \mathrm{mV}-10 \mathrm{mV}-20 \mathrm{mV}, 85$ msec. $B$, Brief stimulation of the siphon nerve evoked a compound EPSP followed closely by inhibitory input (left) in an excitatory interneuron $(I N T)$ at its resting potential $(-55 \mathrm{mV})$. Bath application of 100 $\mu \mathrm{M}$ d-TC blocked all inhibitory input, thus increasing net excitation (right). Interstimulus interval was $5 \mathrm{~min}$. Calibration: $12 \mathrm{mV}, 85 \mathrm{msec}$. $C, A n$ iontophoretic application of $\mathrm{ACh}$ to the cell body of excitatory interneurons (at resting potential) produces a rapid hyperpolarization that reverses near $-60 \mathrm{mV}$ (left). This response is blocked by $100 \mu \mathrm{M}$ d-TC (right). Interstimulus interval was 5 min. Calibration: $3 \mathrm{mV}, 1.3$ sec.

to contain several interneurons such as the L34 excitatory interneurons (Frost, 1987). The L34 neurons have been shown to be directly excited by LE sensory neurons and to produce monosynaptic EPSPs onto LFS motoneurons (Frost, 1987; Frost et al., 1988). They were identified by the presence of these connections (Fig. 4A) and by the characteristic pattern of spontaneous IPSPs and EPSPs that they usually display (Frost, 1987). Compound EPSPs were evoked in these interneurons by siphon nerve stimulation, with an interstimulus interval of $5 \mathrm{~min}$. The cells were always impaled by $\mathrm{K}$-acetate electrodes so as not to modify the $\mathrm{Cl}^{-}$inversion potential, and were not polarized below their resting potential, which was between 50 and $55 \mathrm{mV}$, so as to maintain a physiological electrochemical driving force for $\mathrm{Cl}^{-}$ions. The stimulation intensity was initially adjusted to evoke compound EPSPs that did not produce action potential firing in the interneuron that was impaled. The control recording presented in Figure $4 B$ shows that inhibitory input was always received by the interneuron following the initial excitation. After three to five controls, $100 \mu \mathrm{M}$ d-TC was bath applied. This completely blocked all fast inhibitory input evoked by siphon nerve stimulation in all preparations tested $(n=5)$. A prolonged excitation sometimes accompanied by the generation of action potentials (Fig. $4 B$ ) was noted. The mean increase of the EPSP area was $205.1 \pm 42.8 \%$ above control $(t=7.6, p<0.01 ; n=$ $5)$.
Iontophoresis of ACh onto excitatory interneurons

Although $\mathrm{ACh}$ receptors of both the excitatory (cationic) and inhibitory $\left(\mathrm{Cl}^{-}\right)$types exist in the nervous system of Aplysia, the results presented above suggested that excitatory interneurons involved in the mediation of the GSWR possess predominantly those of the second type. This was verified by directly applying ACh to the cell bodies of excitatory interneurons by iontophoresis. The cells were identified as described above. With the interneurons at their resting potential, a brief $(30-100 \mathrm{msec})$ application of $\mathrm{ACh}$ always produced hyperpolarization $(n=4)$ (Fig. $4 C$ ). The reversal potential $(-58.8 \pm 4.3 \mathrm{mV} ; n=4)$ was consistent with these responses being produced by the opening of $\mathrm{Cl}^{-}$channels. The hyperpolarization was almost totally blocked by $100 \mu \mathrm{M} \mathrm{d}$-TC in all experiments (Fig. $4 C$ ). The average decrease in peak amplitude was $92.8 \pm 1.4 \%(t-7.4, p<0.01)$.

\section{Facilitation of compound EPSCs by connective stimulation in disinhibited preparations}

The identification of significant cholinergic inhibitory control over the excitatory input to motoneurons suggested the possibility that this system is a site of learning-associated modifications. Sensitization of the GSWR could be caused, to a large extent, by a decrease of cholinergic inhibition within the neuronal network. This enhancement of the GSWR may be expressed as dishabituation, potentiation of a previously depressed reflcx, or sensitization, potentiation of a nondepressed reflex. Because the responses in this study were generally minimally depressed in the control period, we refer to the reflex enhancement as sensitization. We started to test this hypothesis in an in vitro analog of sensitization, facilitation of compound EPSCs (excitatory postsynaptic currents) in motoneurons by left connective stimulation (Castellucci et al., 1970; Trudeau and Castellucci, 1992). These experiments were performed with EPSCs instead of EPSPs to prevent complications due to the activation of voltage-dependent conductances during large EPSPs. The motor neurons were voltage clamped with a two-electrode system. It was hypothesized that if sensitization is due in part to a decrease in cholinergic feedback inhibition, relative facilitation will be decreased in preparations disinhibited by nicotinic receptor blockade. Alternatively, if facilitation is caused predominantly by an increase in transmitter release at sensorymotor terminals as well as at the terminals of excitatory interneurons onto motoneurons, then relative facilitation would not be expected to be markedly different in disinhibited systems. Relative facilitation was defined here as the ratio between the area of an EPSC after connective stimulation to that before connective stimulation. Compound EPSCs were evoked in motoneurons of the reflex by a brief electrical stimulation of the siphon nerve through a suction electrode. Two to six control EPSCs were recorded in normal ASW with an interval of $5 \mathrm{~min}$. The interval was $5 \mathrm{~min}$ in the control period to prevent excessive depression of connections in the presence of d-TC and to allow the EPSCs to stay maximally disinhibited. Modulator neurons were then activated by a $5 \mathrm{sec}$ train of stimulation to the left pleuro-abdominal connective ( $5 \mathrm{~V}, 8 \mathrm{~Hz}, 3 \mathrm{msec} /$ shock). Four additional siphon nerve-evoked EPSCs were then recorded with an interval of $1 \mathrm{~min}$. During connective stimulation, the motoneuron was always unclamped so as not to modify the normal spiking activity that these cells display during the facilitating input. Experiments were first performed in normal ASW. Following a $60 \mathrm{~min}$ rest period, $100 \mu \mathrm{M} \mathrm{d}-\mathrm{TC}$ was applied and the 
A_

$\mathrm{MN}$

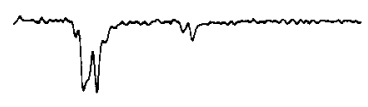

$\sqrt[m]{n^{n+m}} r^{n}$

ASW

$\uparrow$

B_

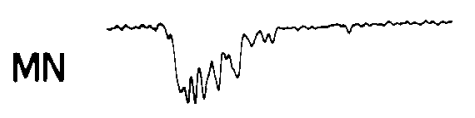

1

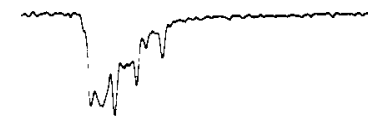
ASW + $d-T C$ $\uparrow$

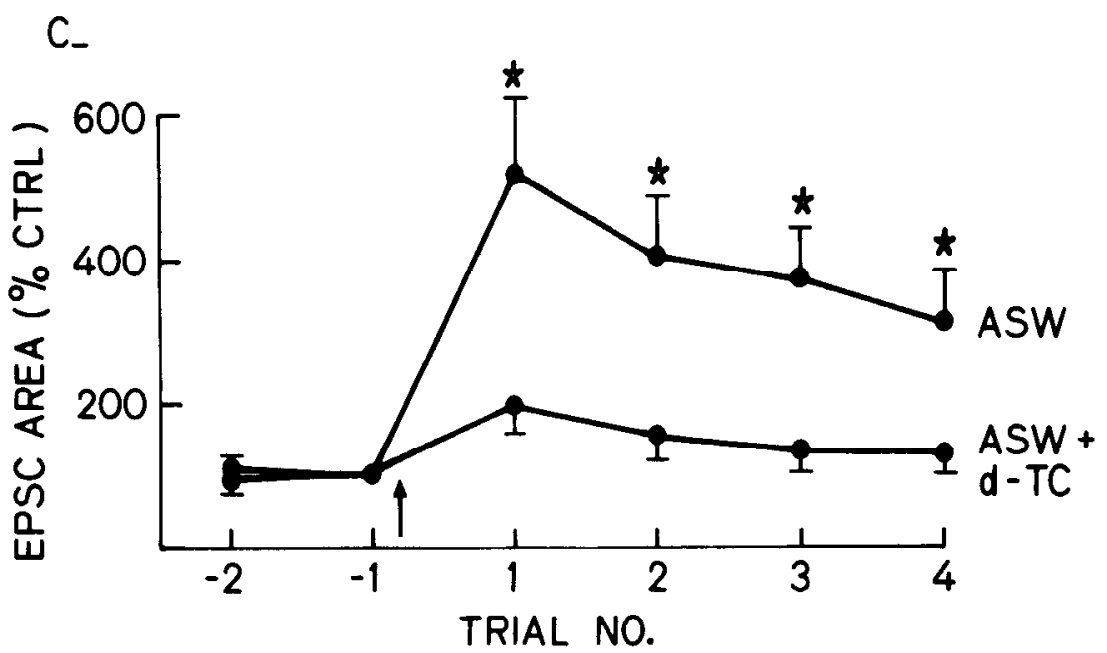

Figure 5. Facilitation in a neuronal network already disinhibited by blocking cholinergic transmission. $A$, Compound postsynaptic currents (EPSCs) were evoked in motoneurons $(M N)$ by stimulation of the siphon nerve. Under control conditions $(A S W)$, activation of modulator neurons by stimulation of the left connective (arrow) significantly potentiated EPSCs (inward current) recorded with the neurons voltage clamped at $-80 \mathrm{mV}$. Interstimulus interval, $1 \mathrm{~min}$. Calibration: $11 \mathrm{nA}, 70$ msec. $B$, The same protocol was also performed in preparations pretreated with $100 \mu \mathrm{M} \mathrm{d}-\mathrm{TC}(A S W+d-T C)$. Calibration: $11 \mathrm{nA}, 70 \mathrm{msec}$. $C$, Summary of the EPSC facilitation data $(n=5)$. The last two controls are plotted along with the four responses recorded after connective stimulation (arrow). Responses are expressed relative to the last plotted control $(-1)$ of each condition. Note that in the presence of $d-T C$, the facilitation of compound EPSCs is reduced. Asterisks indicate significantly different from response recorded in d-TC $(p<0.05)$. preparation allowed to rest for $30 \mathrm{~min}$. Under d-TC, a second facilitation protocol was performed to evaluate the facilitation obtained in disinhibited preparations. The drug was then washed out for 90 min with normal ASW and a third facilitation protocol was performed. The long rest periods were used to allow the system to return to a nonfacilitated state between each facilitation protocol. Results obtained in the two control periods were averaged for statistical comparisons to account for any changes in the efficacy of the facilitation protocols $(n=5)$.

In normal ASW, EPSCs (inward current) were significantly potentiated by this protocol. Figure $5 A$ presents current traces from a representative experiment. The four EPSCs following connective stimulation were significantly different from the last control response $(t=4.0,3.4,3.8,2.8 ; p<0.01,0.01,0.01$, 0.05 ) (Fig. $5 C$ ). In the presence of $100 \mu \mathrm{M}$ d-TC, a significant facilitation of compound EPSCs was also noted (Fig. $5 B$ ); the first EPSC following connective stimulation was significantly different from the last control $(t=2.6, p<0.05)$. The four EPSCs recorded in $\mathrm{d}-\mathrm{TC}$ after the facilitation protocol were each significantly different from their respective counterparts measured in normal ASW $(t=2.9,2.6,3.1,2.3 ; p<0.05)$ (Fig. $5 C$ ). On average, for the four EPSCs measured after connective stimulation, the facilitation obtained in disinhibited preparations was $16.2 \pm 2.4 \%$ of that obtained in normal ASW (301.5 $\pm 43.4 \%$ increase in ASW, $51.6 \pm 15.1 \%$ in d-TC). If the first and second halves (duration) of the last control EPSC were considered separately, the potentiation of the first half in disinhibited networks was $38.4 \pm 6.1 \%$ of that measured in normal ASW (136.3 $\pm 11.8 \%$ in ASW; $54.4 \pm 13.8 \%$ in d-TC), while that of the second half was $4.1 \pm 1.4 \%(818.8 \pm 132.8 \%$ in ASW; $34.4 \pm 17.2 \%$ in $\mathrm{d}-\mathrm{TC}$ ). In these experiments, it was convenient to express the postsynaptic responses as percentages, in relation to the control level of each experiment. This was appropriate because of the variability of the area of EPSCs across different experiments. The data presented above show a decrease in relative facilitation in disinhibited neuronal networks, presumably because disinhibition is part of the mechanism responsible for facilitation. It is possible, however, that a decreasc in relative facilitation may result from a higher initial baseline level, with absolute facilitation unaltered. Absolute facilitation was defined here as the difference between the area of an EPSC after connective stimulation to that before connective stimulation. To exclude this possibility, the compound EPSC data were reanalyzed: the absolute facilitation (increase in EPSC area expressed in $\mathrm{nA} \cdot \mathrm{msec}$ ) of the four responses following connective stimulation was measured in each experiment. The results confirmed that facilitation was less pronounced in preparations disinhibited by d-TC. On average, for the four EPSCs following 
A.
INT
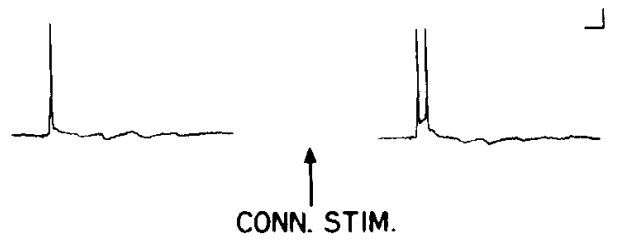

B.

INT
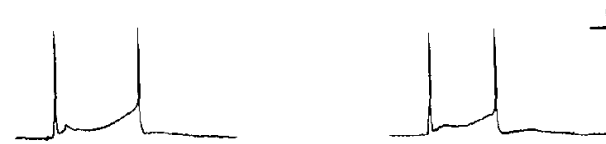

ASW+ $d-T C$

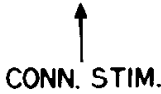

Figure 6. Facilitation of evoked excitatory interneuronal firing. $A$, Under control conditions $(A S W)$, action potential firing of an excitatory interneuron $(I N T)$ evoked by siphon nerve stimulation is potentiated by connective stimulation (CONN. STIM.). Siphon stimulation was adjusted to evoke compound EPSPs of $20-40 \mathrm{mV}$ in motoneurons (not shown). Interstimulus interval, $1 \mathrm{~min}$. Calibration: $10 \mathrm{mV}, 70 \mathrm{msec} . B$, The same protocol was also performed in preparations pretreated with $100 \mu \mathrm{M} \mathrm{d}-T C(A S W+d-T C)$. Note that in the presence of $\mathrm{d}-\mathrm{TC}$, the facilitation of evoked interneuronal firing is reduced. Calibration: 10 $\mathrm{mV}, 70 \mathrm{msec}$

connective stimulation, the absolute increase in EPSC area under d-TC was $55.8 \pm 8.3 \%$ of that obtained in normal ASW $(t$ $=9.3, p<0.01 ; n=5$ ). Analysis of the first and second halves of the EPSCs revealed that absolute facilitation in the presence of d-TC was respectively $139.6 \pm 22.2 \%(t=1.6$, NS $)$ and 12.5 $\pm 4.3 \%(t=8.3, p<0.01)$ of that measured in normal ASW. Facilitation was therefore predominantly prevented in the second component of the EPSCs, that which is controlled by the feedback inhibition.

\section{Facilitation of excitatory interneuronal firing}

The results presented above allow two predictions about the siphon-evoked firing of excitatory interneurons: first, it should be increased following the activation of modulator neurons, and second, liring should not be increased as much by facilitatory protocols when the feedback inhibition these interneurons normally receive has been previously blocked by d-TC. Excitatory interneurons were identified as described above and impaled by a $\mathrm{K}$-acetate electrode. They were left at their resting potential $(50-55 \mathrm{mV})$. The siphon nerve was stimulated every minute at a level sufficient to evoke compound EPSPs with a peak amplitude between 20 and $40 \mathrm{mV}$ in an LFS motoneuron monitored in parallel. In normal ASW, this stimulation initially produced one or two action potentials in the excitatory interneuron. In the presence of d-TC, the same intensity of siphon nerve stimulation initially produced two to seven action potentials. Following three to six control responses, facilitator neurons were activated by a $5 \mathrm{sec}$ train of stimulation to the left pleuroabdominal connective ( $5 \mathrm{~V}, 8 \mathrm{~Hz}, 3 \mathrm{msec} / \mathrm{shock})$. Four additional evoked responses were then recorded. In normal ASW. this treatment significantly increased the evoked firing of excitatory interneurons. While in the last control response the interncurons always fired one action potential, this was increased to an average of $3.0 \pm 0.3$ action potentials (maximal effect) following connective stimulation $(t=6.3, p<0.01)$ (Fig. 6A). The same experiment was repeated after $90 \mathrm{~min}$ in the presence of $100 \mu \mathrm{M} \mathrm{d}-\mathrm{TC}$. In three out of five experiments the protocol was first performed in $\mathrm{d}-\mathrm{TC}$, and then in normal ASW. The last control response in $\mathrm{d}-\mathrm{TC}$ displayed an average of $2.8 \pm 0.4$ action potentials. This was increased to an average of $3.4 \pm 0.6$ after the facilitation protocol $(n=5)$ (Fig. $6 B)$. The increase was not significant.

\section{Facilitation of LE-motoneuron synapses in the presence of d-tubocurarine}

It may be suggested that in preparations pretreated with d-TC, a decrease in the facilitation of compound EPSCs and of evoked excitatory interneuronal firing could be attributed to the direct or indirect blockade of the facilitatory neurons themselves. This possibility was examined by studying the facilitation of monosynaptic LE-motoneuron synapses by left connective stimulation. In cases where synapses displayed multicomponent EPSPs to single action potentials in an LE neuron, only the peak amplitude of the first component was measured. Interstimulus interval was 1 min. After the fourth control EPSP, a 5 sec train of stimulation was applied to the left connective $(5 \mathrm{~V}, 8 \mathrm{~Hz}, 3$ $\mathrm{msec} / \mathrm{shock}$ ), and four additional EPSPs were recorded. Following a $60 \mathrm{~min}$ rest period, $100 \mu \mathrm{M} \mathrm{d}$-TC was applied and the preparation rested for $30 \mathrm{~min}$ more. A second facilitation protocol was then performed in the presence of $\mathrm{d}$-TC. This was done in five preparations, three of these with the reverse order: facilitation in the presence of $\mathrm{d}-\mathrm{TC}, 90 \mathrm{~min}$ washout, and facilitation in normal ASW. The control decline curve from Figure $1 C$ was used for comparison. Figure $7 A$ illustrates recordings from a representative experiment in the presence of d-TC. We found that EPSPs could be efficiently facilitated by connective stimulation performed either in the presence or absence of d-TC. In normal ASW, the first three EPSPs following the facilitation protocol were significantly different from their respective untreated controls $(l=3.7,3.6,3.3 ; p<0.01,0.01,0.05)$. Figure $7 B$ demonstrates that the facilitation measured in normal ASW was not significantly different from that measured in the presence of $100 \mu \mathrm{M} \mathrm{d}-\mathrm{TC}$. Although the first EPSP following connective stimulation in d-TC was significantly different from its nonfacilitated control $(t=2.7, p<0.05 ; n=5)$, it was not significantly different from its facilitated counterpart obtained in normal ASW.

\section{Discussion}

The recent demonstration that approximately $75 \%$ of the evoked afferent input to moloneurons of the GSWR is polysynaptic, together with the finding that this pathway may be selectively modulated (Trudeau and Castellucci, 1992), prompted us to investigate further this component of the neuronal network. It is known that in Aplysia, cholinergic transmission plays a crucial role in many systems. Interestingly, both the feeding and cardiovascular systems are powerfully modulated by multi-action cholinergic interneurons which postsynaptically activate $\mathrm{ACh}$ gated $\mathrm{Cl}^{-}$channels (Tauc and Gerschenfeld, 1962; Blankenship et al., 1971; Gardner, 1971; Segal and Koester, 1982). This type of inhibitory synaptic action could also be involved in the modulation of polysynaptic transmission in the GSWR neuronal network. An example of this type of interneuron in the GSWR network is cholinergic neuron $\mathrm{L} 16$, which is known to produce inhibition in motoneuron L7 as well as in some interncurons (Hawkins et al., 1981a; Frost, 1987). We provide, in this article, an evaluation of the role of cholinergic inhibitory interneurons in the normal mediation and short-term plasticity of the reflex. 
We show that at a concentration that induces a nearly total blockade of cholinergic transmission (Fig. 1), central application of the nicotinic antagonist d-TC markedly potentiates gill contractions evoked by siphon stimulation (Fig. 2). The excitatory input received by motoneurons upon siphon nerve stimulation (Fig. 3) is also considerably prolonged by d-TC. The observation that the initial phase of compound EPSPs is not altered by the drug strongly suggests that the excitatory interneurons themselves are not cholinergic, but that the increased excitation is the result of the blockade of a process that follows the initial excitatory interneuronal firing. This second step is likely to be feedback inhibition upon the excitatory interneurons by cholinergic interneurons that produce inhibition through ACh-gated $\mathrm{Cl}^{-}$channels. The conclusion that d-TC acted at this level was also supported by the finding that a selective blockade of $\mathrm{ACh}$ receptors of the excitatory type by hexamethonium failed to modify compound EPSPs (Fig. 3C). This also confirmed that the excitatory interneurons recruited by siphon afferent input are not themselves cholinergic. One may therefore describe compound EPSPs as being composed of two phases. In the first, excitatory interneuronal firing is strictly determined by the sensory afference and by the intrinsic membrane properties of the excitatory interneurons. In the second phase, an additional influence on excitatory interneuronal firing is the inhibitory input received from inhibitory interneurons that are part of a feedback loop.

More direct support for the mechanism of the prolonged excitation being the uncoupling of feedback inhibition upon excitatory interneurons was obtained by directly recording from excitatory interneurons. Under normal ASW, and at their normal resting potential, it was found that siphon nerve stimulation produced excitation followed closely by strong inhibitory input onto excitatory interneurons (Fig. $4 A$ ). This inhibitory input was totally blocked by d-TC, resulting in an increased excitatory drive. We also showed that excitatory interneurons are directly hyperpolarized by exogenous $\mathrm{ACh}$ applied by iontophoresis, and that this response is blocked by d-TC (Fig. $4 B$ ). These data provide strong support for the effect of d-TC resulting directly from the blockade of cholinergic inhibitory feedback. They render unlikely the involvement of nonspecific effects of d-TC (Nohmi and Kuba, 1984; Capiod and Ogden, 1989). It should be noted, however, that because d-TC has been previously shown to block $\mathrm{Cl}^{-}$channels activated by the iontophoretic application of transmitters other than ACh in the CNS of Aplysia (Carpenter et al., 1977), one cannot be absolutely sure that $\mathrm{ACh}$ is the transmitter at all inhibitory synapses of the GSWR network. It is, however, likely that $\mathrm{ACh}$ is the transmitter because (1) most described fast inhibitory synaptic action in the CNS of Aplysia is known to be cholinergic (this is the case, e.g., at all fast inhibitory synapses that have been characterized in the buccal ganglion), (2) the excitatory interneurons of the GSWR network respond to the iontophoretic application of $\mathrm{ACh}$ by the opening of $\mathrm{Cl}^{-}$channels, (3) biochemical evidence (Giller and Schwartz, 1971) has indicated that there are significant levels of ChAT activity in the section of the abdominal ganglion where we have located (L.-E. Trudeau and V. F. Castellucci, unpublished observations) most of the inhibitory interneurons of the GSWR, and (4) inhibitory interneuron L16, which is part of the GSWR network, has been demonstrated to be cholinergic (Segal and Koester, 1982). Although a blocker such as $\alpha$-bungarotoxin could have been used in an attempt to validate our cholinergic hypothesis further, it should be pointed out that this blocker is
A. $\quad 100 \mu \mathrm{M} \mathrm{d}-\mathrm{TC}$

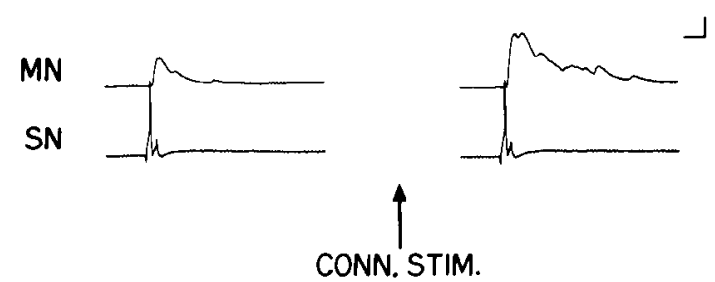

B.

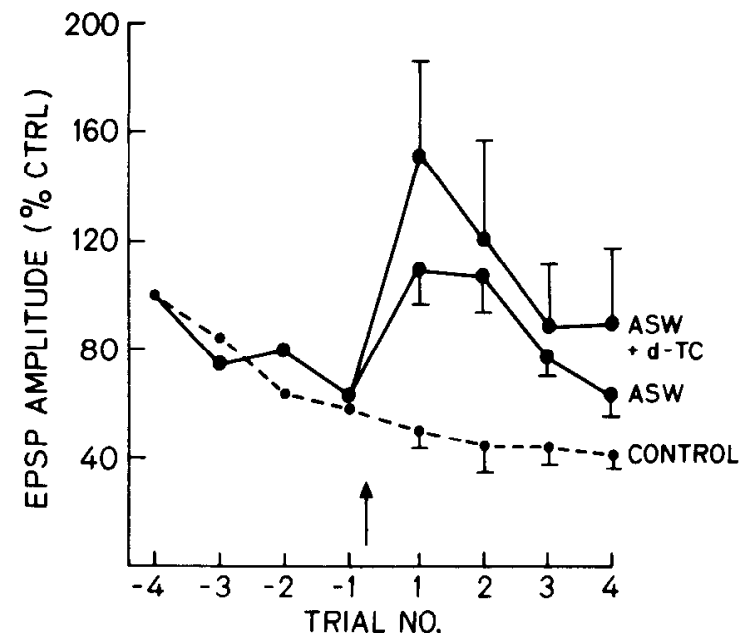

Figure 7. Facilitation of sensory-motor synapses under cholinergic blockade. $A$, EPSPs were evoked in motoneurons $(M N)$ by threshold intracellular stimulation of sensory neurons $(S N)$ of the LE cluster. Interstimulus interval was $1 \mathrm{~min}$. After the fourth EPSP, modulator neurons were activated by stimulation of the left pleuro-abdominal connective. The protocol was performed either in normal ASW or in the presence of $100 \mu \mathrm{M} \mathrm{d}$-TC. The sample traces present the last control (left) and first EPSP recorded following connective stimulation (right) of a representative experiment performed in d-TC. Calibration: $8 \mathrm{mV}-$ $15 \mathrm{mV}, 100 \mathrm{msec}$. B. Summary of the FPSP facilitation data. Arrow indicates connective stimulation following the fourth control EPSP. Note that facilitation was similar in normal ASW and in the presence of d-TC. The control decline curve of Figure $1 C$ is also illustrated for comparison.

also not completely specific in Aplysia CNS (Ono and Salvaterra, 1981; McCaman and Weinreich, 1985).

The importance of such a gating mechanism produced by inhibitory interneurons suggests the possibility that this pathway may be modified during learning in Aplysia. A decreased inhibition could be, for example, a major mechanism of reflex enhancement. This possibility was tested by measuring the facilitation of compound EPSCs by the activation of facilitatory neurons through stimulation of the left pleuro-abdominal connective. This pathway has been previously shown to produce reliable facilitation (Castellucci et al., 1970; Trudeau and Castellucci, 1992). Facilitation was assessed in normal ASW and in the presence of $100 \mu \mathrm{M}$ d-TC. In the latter condition, excitatory interneurons are already released from the inhibitory control of interneurons, and further potentiation of transmission within the GSWR neuronal network cannot result from reduced inhibitory transmission. Relative facilitation of compound EPSC area was found to be smaller in disinhibited preparations (Fig. 5). A more detailed analysis of the facilitation of EPSCs allowed two important observations. First, it was found that facilitation of disinhibited responses was decreased not only in relative 


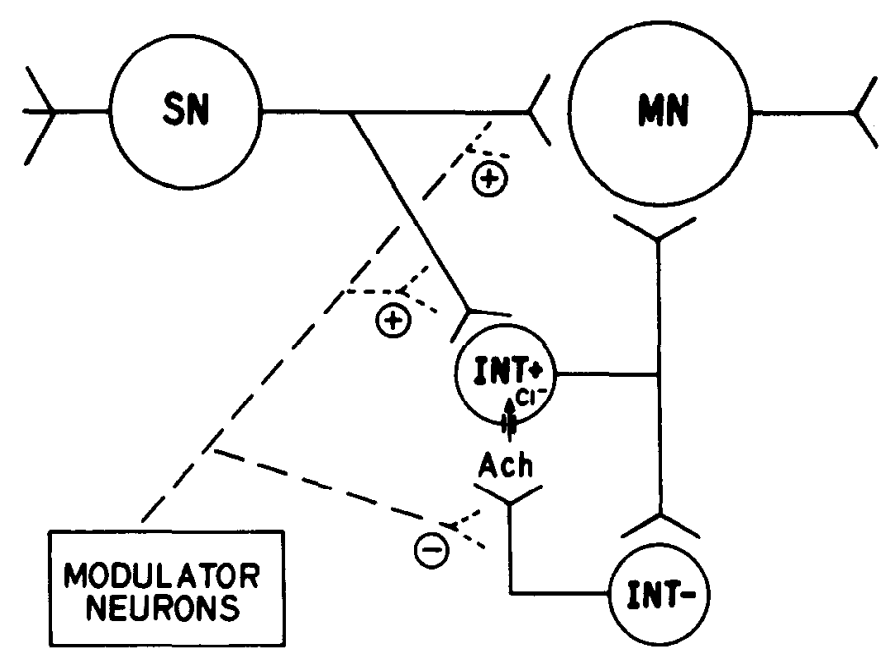

Figure 8. Summary diagram. Simplified diagram to illustrate sites of physiological changes in the GSWR network. Sensory neurons ( $S N)$ monosynaptically excite motoncurons $(M N)$ and excitatory interneurons $\left(I N T^{+}\right)$. The latter synapse upon $M N S$ and inhibitory interneurons $\left(I N T^{-}\right)$, which produce feedback inhibition. The neurons depicted represent populations of similar cells. Sensitization of the GSWR may involve a parallel increase in transmitter release by the sensory neurons, and a reduction of $\mathrm{ACh}$ release by the inhibitory interneurons.

terms, but also in absolute terms. Second, it was observed that the reduced facilitation was predominantly the result of an effect on the sccond component of EPSCs, that which is controlled by the feedback inhibition.

One could argue that a ceiling effect may exist at the level of excitatory interneuronal firing rate. If in disinhibited networks these interneurons already fired at their maximal rate upon siphon stimulation, then one would not expect to see much further potentiation of the afferent input to the motoneurons following connective stimulation. Although this is possible, it only reinforces the idea that excitability of excitatory interneurons is a key site of plasticity for the GSWR, and that cholinergic inhibitory interneurons are an efficient control system of that excitability. This concern was nonetheless directly addressed by recording from excitatory interneurons. It was found (Fig. 6) that these interneurons increased their evoked firing from one to three action potentials following a facilitation protocol performed in normal ASW. In the presence of d-TC, this increase was nonsignificant: cvokcd firing changed from an avcrage of 2.8 to an average of 3.4 after facilitation. These results confirm the conclusions obtained with compound postsynaptic responses recorded in motoneurons. They also render unlikely, under our experimental conditions, the existence of a ceiling effect at the level of excitatory interneuronal firing rate, because these interneurons display very little accommodation and are easily capable of firing at a higher frequency. Finally, the finding that, in the presence of d-TC, monosynaptic connections between LE sensory neurons and motoneurons of the reflex may still be efficiently potentiated by left connective stimulation (Fig. 7) indicated that the decreased facilitation observed in the presence of $\mathrm{d}-\mathrm{TC}$ is not caused by preventing the facilitatory neurons from being activated or functional. This conclusion is also supported by the finding that even though facilitation is reduced in disinhibited preparations, it is not abolished: significant facilitation remains (Figs. 5,6), and may in all likelihood be attributed to increased transmitter release at sensory neuron terminals synapsing onto motoneurons (Castellucci et al., 1970, 1980; Klein et al., 1982; Hochner et al., 1986a; Goldsmith and Abrams, 1991), and also possibly at the terminals of excitatory interneurons onto motoneurons. The demonstration of normal facilitation of monosynaptic LE-motoneuron synapses in the presence of d-TC was thought important in the light of the recent finding that d-TC has antagonistic properties at some types of receptors for 5-HT (Malone et al., 1991), a neuromodulator thought to be involved in sensitization in Aplysia. One may even note in Figure 7 a tendency toward an increased facilitation of monosynaptic connections in the presence of d-TC. Although nonsignificant, this tendency may suggest that facilitatory interneurons are themselves under feedback inhibitory control. In this regard, it has already been shown that the identified L29 facilitator neurons, thought to be involved in heterosynaptic facilitation of sensory-motor connections, do receive feedback inhibition (Hawkins et al., 1981a; Frost, 1987) from interneurons L 30 and L16. It is known that inhibition produced by L16 is cholinergic (Segal and Koester, 1982).

Although a small number of inhibitory interneurons have been previously identified in the GSWR neuronal network (Hawkins et al., 1981a,b; Frost, 1987; Frost et al., 1988), few data have been reported as to their relative importance in the mediation of the reflex and their functional role and plastic properties. The data presented here suggest that cholinergic inhibitory interneurons play a crucial role in the gating of the sensory input to the motoneurons. The mechanism of this gating appears to be a powerful feedback inhibition onto excitatory interncurons intercalated between sensory and motor neurons. Because the same results were obtained with LFS motoneurons, which control siphon contractions, and with motoneuron L7, which participates in triggering gill contractions, the same type of inhibitory circuits appear to control the evoked output to both these organs. The data also suggest that a functional uncoupling or diminution of cholinergic inhibitory interneuronal transmission may be a mechanism for the facilitation of the GSWR observed during sensitization, a nonassociative form of learning. Recent data from Fischer and Carew (1993) also suggest that an opposite modulation of inhibitory transmission may be involved in short-term decrements of the reflex. The involvement of disinhibitory mechanisms in dishabituation remains to be explored; our preliminary results indicate that they are involved as well. We have recently shown that the neuropeptide $\mathrm{SCP}_{\mathrm{B}}$, present in the nervous system of Aplysia (Abrams et al., 1984; Lloyd et al., 1985), is able to potentiate transmission in the GSWR neuronal network through a preferential action on the polysynaptic component of the circuit (Trudeau and Castellucci, 1992). An interesting possibility, which we are currently pursuing, is that $\mathrm{SCP}_{\mathrm{B}}$ may cause a reduction of $\mathrm{ACh}$ release by some inhibitory interneurons. A simplified diagram of the GSWR neuronal network is illustrated in Figure 8. It suggests that an activation of modulatory neurons may simultaneously lead to an enhancement of transmitter release at sensory neuron terminals, and to a decrease of $\mathrm{ACh}$ release at the terminals of interneurons involved in feedback inhibition. Both mechanisms, acting in parallel, may be necessary to increase the firing rate of excitatory interneurons and optimally sensitize the withdrawal reflex. Work is in progress, in our laboratory, to localize and further characterize the behavior of these cholinergic interneurons during various forms of learning in Aplysia. The possible involvement of cholinergic disinhibitory processes in long-term memory will also be considered. 


\section{References}

Abrams TW, Castellucci VF, Camardo JS, Kandel ER, Lloyd PE (1984) Two endogenous neuropeptides modulate the gill and siphon withdrawal reflex in Aplysia by presynaptic facilitation involving cAMPdependent closure of a serotonin-sensitive potassium channel. Proc Natl Acad Sci USA 81:7956-7960.

Bailey CH, Chen M, Keller F, Kandel ER (1992) Serotonin-mediated endocytosis of apCAM: an early step of learning-related synaptic growth in Aplysia. Science 256:645-649.

Baux G, Fossier P, Tauc L (1990) Histamine and FLRFamide regulate acetylcholine release at an identified synapse in Aplysia in opposite ways. J Physiol (Lond) 429:147-168.

Blankenship JE, Wachtel H, Kandel ER (1971) Ionic mechanisms of excitatory, inhibitory, and dual synaptic actions mediated by an identified interneuron in the abdominal ganglion of Aplysia. J Neurophysiol 34:76-92.

Brunelli M, Castellucci VF, Kandel ER (1976) Synaptic facilitation and behavioral sensitization in Aplysia: possible role of serotonin and cAMP. Science 194:1178-1180.

Byrne JH (1983) Identification and initial characterization of a cluster of command and pattern-generating neurons underlying respiratory pumping in Aplysia californica. J Neurophysiol 49:491-507.

Capiod T, Ogden DC (1989) The properties of calcium-activated potassium channels in guinea-pig isolated hepatocytes. J Physiol (Lond) 409:285-295.

Carew TJ, Pinsker H, Rubinson K, Kandel ER (1974) Physiological and biochemical properties of neuromuscular transmission between identified motoneurons and gill muscle in Aplysia. J Neurophysiol 37:1020-1040.

Carpenter DO, Swann JW, Yarowsky PJ (1977) Effect of curare on responses to different neurotransmitters in Aplysia neurons. J Neurobiol 8:119-132.

Castellucci VF, Pinsker H, Kupfermann I, Kandel ER (1970) Neuronal mechanisms of habituation and dishabituation of the gill-withdrawal reflex in Aplysia. Science 167:1745-1748.

Castellucci VF, Kandel ER, Schwartz JH, Wilson FD, Nairn AC, Greengard P (1980) Intracellular injection of the catalytic subunit of cyclic AMP-dependent protein kinase stimulates facilitation of transmitter release underlying behavioral sensitization in Aplysia. Proc Natl Acad Sci USA 77:7492-7496.

Dash PK, Hochner B, Kandel ER (1990) Injection of the cAMPresponsive element into the nucleus of Aplysia sensory neurons blocks long-term facilitation. Nature 345:718-721.

Davis WJ, Gillette R, Kovac MP, Croll RP, Matera EM (1983) Organization of synaptic inputs to paracerebral feeding command interneurons of Pleurobranchaea californica. III. Modifications induced by experience. J Neurophysiol 49:1557-1572.

Elaagouby A, Ravel N, Gervais R (1991) Cholinergic modulation of excitability in the rat olfactory bulb: effect of local application of cholinergic agents on evoked field potentials. Neuroscience 45:653662.

Fischer TM, Carew TJ (1993) Activity-dependent potentiation of recurrent inhibition: a mechanism for dynamic gain control in the siphon wilhdrawal reflex of Aplysia. J. Neurosci 13:1302-1314.

Frazier WT, Kandel ER, Kupfermann I, Waziri R, Coggeshall RE (1967) Morphological and functional properties of identified neurons in the abdominal ganglion of Aplysia californica. J Neurophysiol 30: 1288-1351.

Frost WN (1987) Mechanisms contributing to short- and long-term sensitization in Aplysia. PhD thesis, Columbia University, New York.

Frost WN, Clark GA, Kandel ER (1988) Parallel processing of shortterm memory for sensitization in Aplysia. J Neurobiol 19:297-334.

Gardner D (1971) Bilateral symmetry and interneuronal organization in the buccal ganglia of Aplysia. Science 173:550-553.

Giller E Jr, Schwartz JH (1971) Choline acetyltransferase in identified neurons of abdominal ganglion of Aplysia californica. J Neurophysiol 34:93-115.
Goldsmith BA, Abrams TW (1991) Reversal of synaptic depression by serotonin at Aplysia sensory neuron synapses involves activation of adenylyl cyclase. Proc Natl Acad Sci USA 88:9021-9025.

Hawkins RD, Castellucci VF, Kandel ER (1981a) Interneurons involved in mediation and modulation of gill-withdrawal reflex in Aplysia. I. Identification and characterization. J Neurophysiol 45:304314.

Hawkins RD, Castellucci VF, Kandel ER (1981b) Interneurons involved in mediation and modulation of the gill-withdrawal reflex in Aplysia. II. Identified neurons produce heterosynaptic facilitation contributing to behavioral sensitization. J Neurophysiol 45:315-326.

Hochner B, Klein M, Schacher S, Kandel ER (1986a) Action-potential duration and the modulation of transmitter release from the sensory neurons of Aplysia in presynaptic facilitation and behavioral sensitization. Proc Natl Acad Sci USA 83:8410-8414.

Hochner B, Klein M, Schacher S, Kandel ER (1986b) Additional component in the cellular mechanism of presynaptic facilitation contributes to behavioral dishabituation in Aplysia. Proc Natl Acad Sci USA 83:8794-8798.

Kehoe J (1972) Three acetylcholine receptors in Aplysia neurones. J Physiol (Lond) 225:115-146.

Klein M, Camardo J, Kandel ER (1982) Serotonin modulates a specific potassium current in the sensory neurons that show presynaptic facilitation in Aplysia. Proc Natl Acad Sci USA 79:5713-5717.

Korn H, Oda Y, Faber DS (1992) Long-term potentiation of inhibitory circuits and synapses in the central nervous system. Proc Natl Acad Sci USA 89:440-443.

Lederhendler II, Gart S, Alkon DL (1986) Classical conditioning of Hermissenda: origin of a new response. J Neurosci 6:1325-1331.

Lloyd PE, Mahon AC, Kupfermann I, Cohen JL, Scheller RH, Weiss KR (1985) Biochemical and immunocytological localization of molluscan small cardioactive peptides in the nervous system of Aplysia californica. J Neurosci 5:1851-1861.

Lukowiak K, Peretz B (1977) The interaction between the central and peripheral nervous systems in the mediation of gill withdrawal reflex behavior in Aplysia. J Comp Physiol 117:219-244.

Malone HM, Peters JA, Lambert JJ (1991) Physiological and pharmacological propertics of 5-HT3 receptors: a patch-clamp study. Neuropeptides 19:25-30.

Mayford M, Barzilai A, Keller F, Schacher S, Kandel ER (1992) Modulation of an NCAM-related adhesion molecule with long-term synaptic plasticity in Aplysia. Science 256:638-644.

McCaman RE, Weinreich D (1985) Histaminergic synaptic transmission in the cerebral ganglion of Aplysia. J Neurophysiol 53:10161037.

Montarolo PG, Goelet P, Castellucci VF, Morgan J, Kandel ER (1986) A critical time-window for macromolecular synthesis in long-term heterosynaptic facilitation in Aplysia. Science 234:1249-1254.

Moot DD, Lewis DV (1991) Facilitation of the induction of long-term potentiation by $\mathrm{GABA}_{\mathrm{B}}$ receptors. Science 252:1718-1720.

Nohmi M, Kuba K (1984) (+)-Tubocurarine blocks the $\mathrm{Ca}^{++}$-dependent $\mathrm{K}^{+}$channel of the bullfrog ganglion cell. Brain Res 301:146148.

Ono JK, Salvaterra PM (1981) Snake $\alpha$-toxin effects on cholinergic and noncholinergic responses of Aplysia californica neurons. J Neurosci $1: 259-270$.

Segal MM, Koester J (1982) Convergent cholinergic neurons produce similar postsynaptic actions in Aplysia: implication for neural organization. J Neurophysiol 47:742-759.

Tauc L, Gerschenfeld HM (1962) A cholinergic mechanism of inhibitory synaptic transmission in a molluscan nervous system. J Neurophysiol 25:236-262.

Trudeau L-E, Castellucci VF (1992) Contribution of polysynaptic pathways in the mediation and plasticity of Aplysia gill and siphon withdrawal reflex: evidence for differential modulation. J Neurosci 12:3838-3848.

Weiss S, Goldberg JI, Edstrom JP, Lukowiak K (1984) Cholinergic receptors in the Aplysia gill. J Neurobiol 15:325-332. 